\title{
Hallazgos tomográficos en afectación pulmonar por COVID-19, experiencia inicial en el Instituto Nacional de Enfermedades Respiratorias Ismael Cosío Villegas, Ciudad de México
}

\section{CT findings in COVID-19 lung disease, initial experience at Instituto Nacional de Enfermedades Respiratorias Ismael Cosío Villegas, Ciudad de México}

\author{
Fortunato Juárez-Hernández, ${ }^{*}$ Marina Patricia García-Benítez, ${ }^{*}$ \\ Alda Marcela Hurtado-Duarte, ${ }^{*}$ Randall Rojas-Varela, ${ }^{*}$ Juan Pablo Farías-Contreras, ${ }^{*}$ Lya Edith Pensado Piedra, ${ }^{*}$ \\ Aloisia Paloma Hernández Moralesa, ${ }^{*}$ Roberto Sotelo Robledo, ${ }^{*}$ Julio César Gómez Penagos*
}

*Instituto Nacional de Enfermedades Respiratorias Ismael Cosío Villegas, Ciudad de México, México.

RESUMEN. Introducción: La pandemia por SARS-CoV-2 es un importante problema de salud pública a nivel mundial, con altas tasas de morbimortalidad. La tomografía computarizada es fundamental en el proceso diagnóstico dada su alta sensibilidad. Objetivo: Describir los hallazgos tomográficos en la afectación pulmonar por COVID-19. Material y métodos: Estudio transversal analítico. Pacientes atendidos en el Instituto Nacional de Enfermedades Respiratorias, Ciudad de México, con definición operacional vigente de caso sospechoso para COVID-19, que contaran con prueba de RT-PCR específica y tomografía computarizada de tórax en la valoración inicial. Se realizó un análisis descriptivo y analítico mediante la prueba de $\chi^{2}$ y t de Student. Se empleó el programa Epi-Info versión 7. Resultados: Se analizaron 56 pacientes, con una edad promedio de 51 años, 61\% fue del sexo masculino. $52 \%$ presentaron comorbilidades, siendo la diabetes mellitus la más frecuente. Los síntomas que principalmente se observaron fueron fiebre, tos y cefalea. El patrón tomográfico que predominó fue mixto, con localización subpleural y bilateral. Conclusión: El espectro tomográfico pulmonar de la población estudiada se caracterizó por dos patrones principales: tipo mixto (áreas de consolidación con vidrio deslustrado) y empedrado.

Palabras clave: Neumonía viral, SARS-CoV-2, COVID-19, tomografía computarizada, INER.

\section{INTRODUCCIÓN}

Desde el año 2002, posterior a la epidemia por el síndrome respiratorio agudo severo (SARS, por sus siglas en inglés) se han descubierto múltiples coronavirus asociados

Correspondencia:

Dr. Fortunato Juárez-Hernández

Instituto Nacional de Enfermedades Respiratorias Ismael Cosío

Villegas, Ciudad de México.

Correo electrónico: drjuarez.radiologo@gmail.com

Trabajo recibido: 07-V-2020; aceptado: 26-V-2020.
ABSTRACT. Introduction: The SARS-CoV-2 pandemic is a major public health problem worldwide, with high morbidity and mortality rates. Computed tomography (CT) is essential in the diagnostic process given its high sensitivity. Objective: To describe the tomographic findings in COVID-19 lung involvement. Material and methods: Analytical crosssectional study. Patients attended at the INER, CDMX, with a current operational definition of a suspected case for COVID-19, who had a specific RT-PCR test and chest CT in the initial evaluation. A descriptive and analytical analysis was performed using the Student's $\chi^{2}$ and t tests. The Epi-Info version 7 program was used. Results: 56 patients were analyzed, with an average age of 51 years, $61 \%$ were male. $52 \%$ presented comorbidities, with diabetes mellitus being the most frequent. The symptoms that were mostly observed were fever, cough and headache. The tomographic pattern that predominated was mixed, with a subpleural and bilateral location. Conclusion: The pulmonary tomographic spectrum of the studied population was characterized by two main patterns: mixed type (areas of ground glass consolidation) and crazy paving.

Keywords: Pneumonia viral, SARS-CoV-2, COVID-19, computed tomography, INER.

a esta entidad. En la actualidad, la afectación pulmonar por COVID-19 es ocasionada por un patógeno humano y animal perteneciente a la familia del betacoronavirus y específicamente del subgénero de virus del SARS que le otorga el nombre de SARS-CoV-2.

Este patógeno fue causante de un foco de neumonía «atípica» en la ciudad de Wuhan, provincia de Hubei, China en el mes de diciembre de 2019. Esta manifestación clínica tuvo una rápida diseminación nacional y mundial que condicionó la actual pandemia, ${ }^{2}$ contabilizando al 23 de abril de 2020 más de 2,500,000 personas infectadas a nivel mundial y más de 175,000 muertes. No obstante, 
en México fue el pasado 27 de febrero cuando se registró el primer caso de coronavirus en el país; y en tan sólo dos meses, este virus logró infectar a 9,501 personas produciendo la muerte asociada a COVID-19 de 857 personas. ${ }^{3}$

De manera general, en el caso de las neumonías virales se ha determinado que las características en radiografía convencional de tórax son dependientes del huésped (edad, morbilidades, estado inmunológico y nutricional), demostrando que hasta en 58\% de los casos ésta puede resultar normal. Dentro de los posibles hallazgos se destacan: áreas de consolidación, opacidades nodulares, engrosamiento de paredes bronquiales y derrame pleural; además, puede ocurrir una rápida progresión hacia síndrome de distrés respiratorio agudo., ${ }^{4,5}$

Conforme avanza el conocimiento de estas enfermedades virales y aunado a las mejoras tecnológicas en imagen diagnóstica, se ha demostrado que estos datos radiográficos cuando son evidenciados en tomografía de tórax pueden brindar un acercamiento etiológico viral con base en su morfología y distribución, ${ }^{6}$ por lo que en este método de imagen podemos observar cinco grandes hallazgos: alteraciones en la atenuación parenquimatosa, vidrio deslustrado, opacidades nodulares, engrosamiento de septos interlobulillares y de paredes bronquiales, también pueden existir otros hallazgos asociados según el ente patológico existente. ${ }^{5}$

En el caso del coronavirus, muchos de los hallazgos tomográficos han mostrado afectación del parénquima pulmonar con densidades en vidrio deslustrado de distribución periférica y bilateral. ${ }^{7}$ También se ha observado que la evolución de estos hallazgos tomográficos pulmonares por COVID-19 depende de la cantidad de días de sintomatología, determinándose que en los primeros días de esta afección, específicamente entre 0 y cuatro días, hasta $17 \%$ de los estudios son normales; no obstante; las principales alteraciones son vidrio deslustrado con distribución multisegmentaria y periférica. Después, entre los cinco y 13 días estos hallazgos pueden progresar, también puede existir patrón en empedrado hasta en 19\% de los casos y aparición o aumento de consolidaciones. Por último, a partir de los 14 días hay grados variables de los hallazgos previamente descritos y podría ocurrir una resolución de estos cambios en alrededor de 26 días. ${ }^{8}$

Dado lo anterior, la tomografía computarizada (TC) de tórax se ha considerado parte fundamental en el proceso de diagnóstico para la afección pulmonar por SARS-CoV-2 debido a su alta sensibilidad en la detección de cambios parenquimatosos compatibles con la enfermedad (hasta $88 \%$ ); ${ }^{7}$ y también al ser ésta una herramienta accesible en muchos de nuestros centros hospitalarios de segundo y tercer nivel. Debemos entender su valor diagnóstico ante la actual pandemia.

\section{Fisiopatología}

El SARS-Cov-2 es un virus ARN de una sola cadena, con una similitud genética de cerca de $80 \%$ con el SARS-
CoV- $1^{1}$ y de $50 \%$ con el MERS-CoV. ${ }^{9}$ El período de incubación ronda los 14 días, expresándose el inicio de los síntomas en la mayoría de los casos entre cuatro y cinco días posterior a la exposición. ${ }^{2}$

Asimismo, se ha correlacionado su entrada celular a través de los receptores de la enzima convertidora de angiotensina $2,{ }^{1,9}$ por lo que su interacción puede manifestarse en múltiples tejidos corporales. A nivel pulmonar, pese a no estar del todo clara su fisiopatología en este momento, su ingreso celular condiciona daño alveolar difuso con exudados celulares fibromixoides, descamación de neumocitos y formación de membrana hialina como posibles vías de afectación. ${ }^{10}$

Además, tampoco existe una evidencia real en la función de los mediadores inflamatorios en la patología pulmonar; sin embargo, por su gran similitud genética con los patógenos mencionados hay correlación de la gravedad de la enfermedad debido a la respuesta inflama-

Tabla 1: Descripción de aspectos sociodemográficos, epidemiológicos y clínicos de casos sospechosos de COVID-19.

\begin{tabular}{|l|r|}
\hline Variables & $\mathrm{n}=56$ \\
\hline Características sociodemográficas & \\
Edad (años) & $51 \pm 13$ \\
Masculino & $34(61)$ \\
Residente de Ciudad de México & $53(95)$ \\
Comorbilidades presentes & $29(52)$ \\
*Tipos de comorbilidades & \\
$\cdot \quad$ Diabetes mellitus & $18(62)$ \\
$\cdot \quad$ Hipertensión arterial & $12(41)$ \\
. Obesidad $\quad$ Otros & $6(21)$ \\
Características epidemiológicas & $7(24)$ \\
Caso COVID-19 & \\
Contacto con caso positivo COVID-19 & $25(45)$ \\
Contacto con caso sospechoso de COVID-19 & $9(16)$ \\
Historia de viaje & $7(13)$ \\
Lugares turísticos & $15(27)$ \\
$\quad$ Internacional & \\
• Nacional & $5(33)$ \\
Características de la enfermedad & $10(67)$ \\
Duración de la enfermedad (días), & \\
Fiebre & $85 \pm 68$ \\
Tos Cefalea & $17(30)$ \\
Disnea & $51(91)$ \\
Dolor pleurítico & $32(75)$ \\
Saturación de oxígeno & $38(68)$ \\
Intubados & \\
\hline
\end{tabular}

Variables cualitativas expresadas en frecuencia (\%); variables cuantitativas expresadas en promedio \pm desviación estándar (DE).

*Algunos pacientes presentaron hasta tres comorbilidades. 

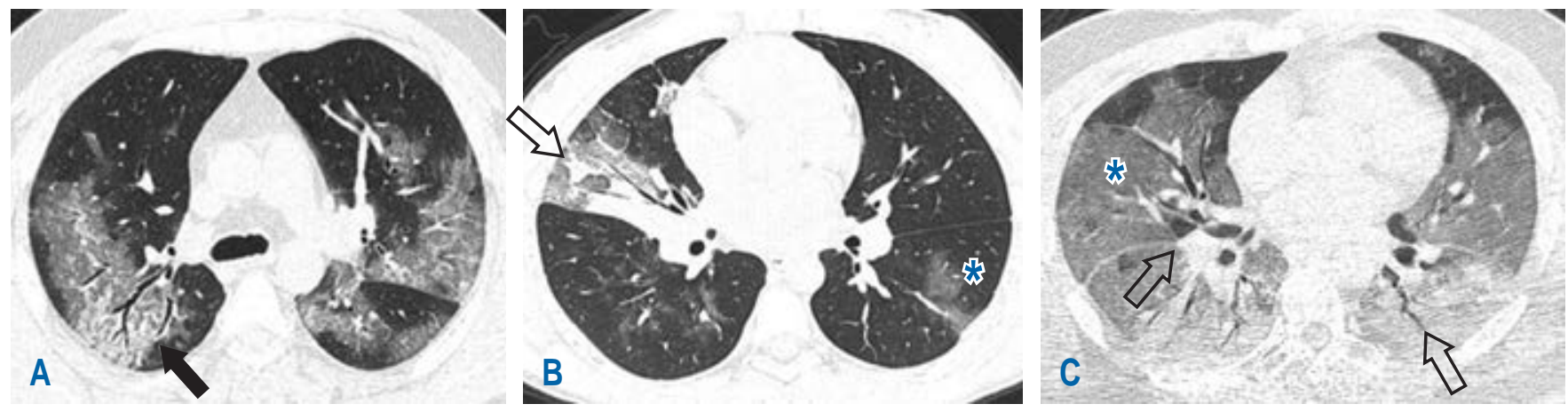

Figura 1: Tomografía computarizada de tórax en ventana pulmonar; patrón mixto. A) Vidrio deslustrado bilateral con tendencia a la consolidación en lóbulo inferior derecho (flecha negra). B) En lóbulo medio se identifica área de consolidación (flecha hueca) y parches de vidrio deslustrado en lóbulos inferiores( $\left.{ }^{*}\right)$. C) Existen zonas de consolidación en lóbulos inferiores asociados a extensa área en vidrio deslustrado (*).

toria desencadenada ya conocida en ellos (elevación de quimiocinas y de interferón tipo II así como incremento de citoquinas e interleuquinas, estas últimas determinan su severidad). ${ }^{11}$

\section{Objetivo general}

Describir los hallazgos tomográficos en la afectación pulmonar por COVID-19 en la población mexicana.

\section{MATERIAL Y MÉTODOS}

Se realizó un estudio transversal y descriptivo en pacientes con neumonía por COVID-19, que fueron atendidos en el Servicio de Urgencias del Instituto Nacional de Enfermedades Respiratorias (INER), Ismael Cosío Villegas en la Ciudad de México. De acuerdo con los criterios de inclusión fueron seleccionados todos los casos que cumplieron con la definición operacional de caso sospechoso de COVID-19, «personas de cualquier edad que en los últimos siete días hayan presentado al menos dos de los siguientes signos y síntomas: tos, fiebre o cefalea (dolor de cabeza intenso y persistente) acompañadas de al menos uno de los siguientes signos o síntomas: dificultad para respirar, falta de apetito, dolor de articulaciones o músculos, conjuntivitis, dolor de garganta y congestión nasal», de acuerdo a los lineamientos de la Secretaría de Salud, con toma de prueba de RT-PCR para SARS-CoV-2 y adquisición de estudio tomográfico de tórax en la valoración inicial. Se excluyeron los casos con estudio tomográfico de tórax con artefacto de movimiento que impidiera su lectura. El Comité de Ética del INER aprobó este estudio y otorgó una excepción del consentimiento informado de los participantes del estudio. La confidencialidad de los datos del paciente se protegió mediante la asignación de un número de identificación y los datos electrónicos se almacenaron en una computadora bloqueada con contraseña.

\section{Procedimiento}

Los datos clínicos utilizados en este estudio se tomaron de la información registrada en el expediente electrónico, lo cual se realizó a través de una ficha estructurada donde se obtuvieron datos sociodemográficos, epidemiológicos y clínicos de los pacientes. Para confirmar la positividad de los casos se les realizó muestra de hisopado nasofa-
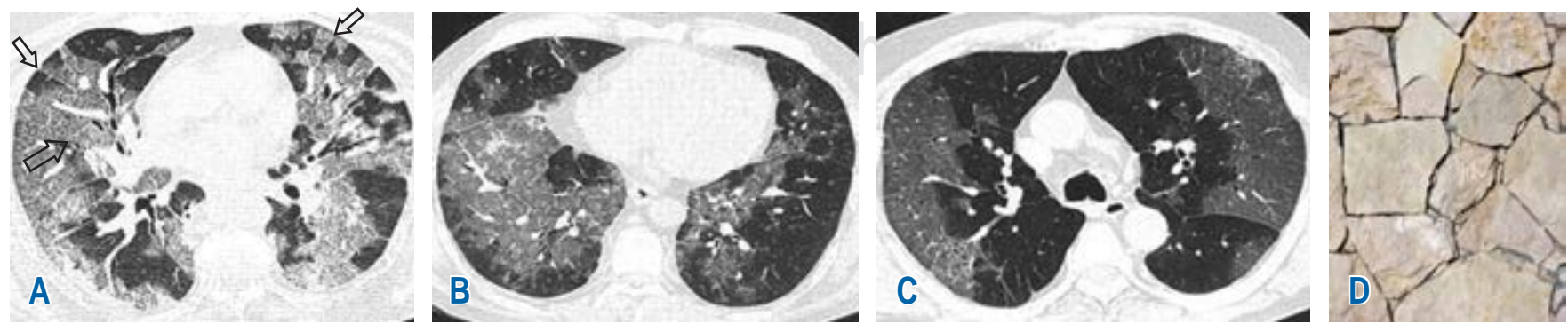

Figura 2: Tomografía computarizada de tórax en ventana pulmonar, A) patrón en empedrado, el cual consiste en vidrio deslustrado asociado con engrosamiento de los septos inter y/o intralobulillares (flechas huecas), lo cual le confiere un aspecto en «empedrado" también conocido como Crazy Paving (imagen D); B) empedrado de distribución central; C) patrón en empedrado de distribución clásicamente subpleural. 

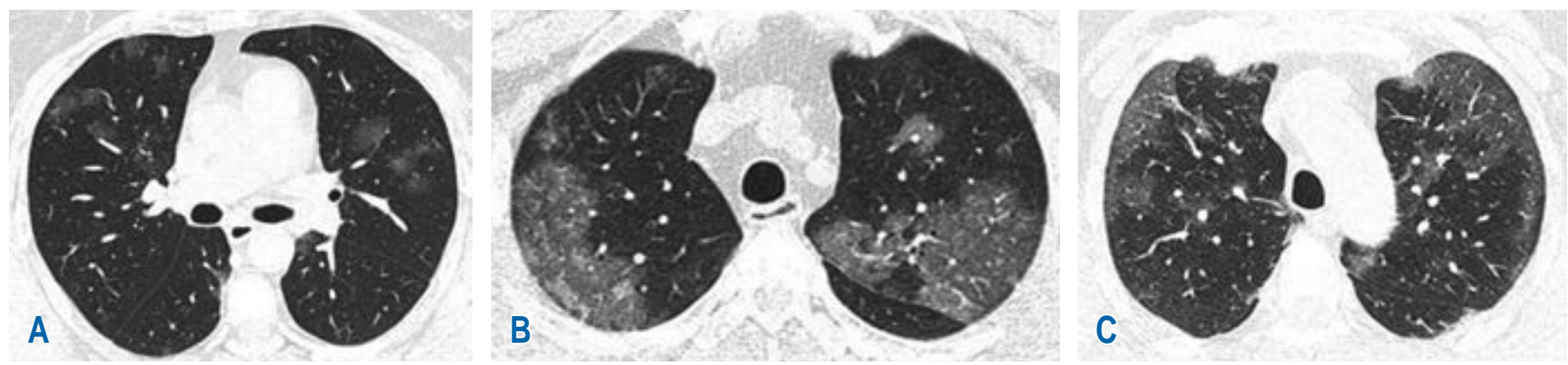

Figura 3: Tomografía computarizada de tórax en ventana pulmonar, patrón en vidrio deslustrado. A) Distribución parcheada de las lesiones en lóbulos superiores. B y C) Incremento de la atenuación en vidrio deslustrado de distribución subpleural.

ríngeo o lavado bronquioloalveolar (LBA), con prueba de laboratorio de transcriptasa inversa en tiempo real (RT-PCR); además, a todos los casos se les hizo panel para infecciones virales integrada por la evaluación de 10 patógenos respiratorios. Al ingreso hospitalario se elaboró TC de tórax y los resultados del estudio fueron revisados en el sistema PACS institucional por tres radiólogos con experiencia en la interpretación de imágenes torácicas con 20, 16 y cinco años de experiencia, los cuales describieron las principales características de la TC con base en el vocabulario propuesto por el Glosario Fleischner 2008, ${ }^{12}$ (patrón pulmonar, localización -unilateral o bilateral-, afectación por lóbulo, distribución y otros hallazgos).

\section{Protocolo de TC de tórax}

Los estudios se realizaron en un tomógrafo multidetector marca SIEMENS Sensation de 64 cortes, con software Syngo CT 2014A, el paciente se colocó en posición supina, en inspiración máxima, con adquisición volumétrica, en ventana pulmonar y mediastinal. Los principales parámetros de escaneo fueron los siguientes: voltaje del tubo $=120$ $k V p$, con modulación automática de la corriente del tubo (70-120 mAs), Pich de 1, espesor de corte de $1 \mathrm{~mm}$ y matriz de $512 \times 512$. Todas las imágenes fueron reconstruidas con un algoritmo de alta resolución espacial.

\section{Análisis estadístico}

El análisis descriptivo de las variables cualitativas se expresó en frecuencias y porcentajes. Las variables cuantitativas se expresaron en media, desviación estándar y rango.

Se hizo una comparación entre proporciones mediante la prueba de $\chi^{2}$ o la prueba exacta de Fisher y la prueba $t$ de Student para comparación entre medias, tomando como significativo el valor de $p<0.05$. El análisis de los datos se realizó en el programa Epi-Info versión 7.

\section{RESULTADOS}

Se analizaron 56 pacientes que fueron atendidos en el Servicio de Urgencias del INER en la Ciudad de México. La edad promedio fue de 51 años. ${ }^{13}$ El sexo masculino tuvo mayor afectación con 61\%. 95\% eran residentes de la Ciudad de México. Entre la población estudiada 52\% tenían antecedentes de al menos una comorbilidad, siendo la diabetes mellitus más frecuente con $62 \%$.

$45 \%$ (25) de los casos estudiados contaron con resultado positivo a la prueba RT-PCR, 34\% estaba pendiente de resultado y el resto se reportó negativo (12). En 16\% se documentó antecedentes de contacto con caso confirmado de COVID-19, todos residentes de la Ciudad de México. 27\% refirieron viajes en los últimos 15 días con destino interna-
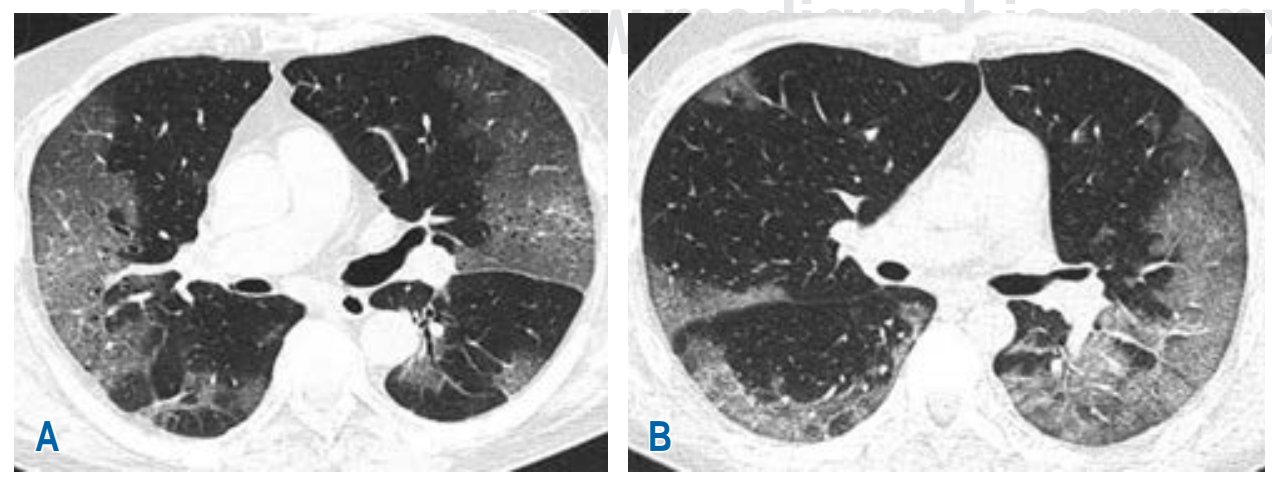

Figura 4:

Tomografía computarizada de tórax en ventana pulmonar. $\mathbf{A}$ y $\mathbf{B}$ ) áreas extensas en vidrio deslustrado y patrón en empedrado asociado, la afectación es de predominio subpleural bilateral. 
Figura 5:

Tomografía computarizada de tórax en ventana pulmonar. A y B) Muestran lesiones en vidrio deslustrado, distribución en parches y bilateral.
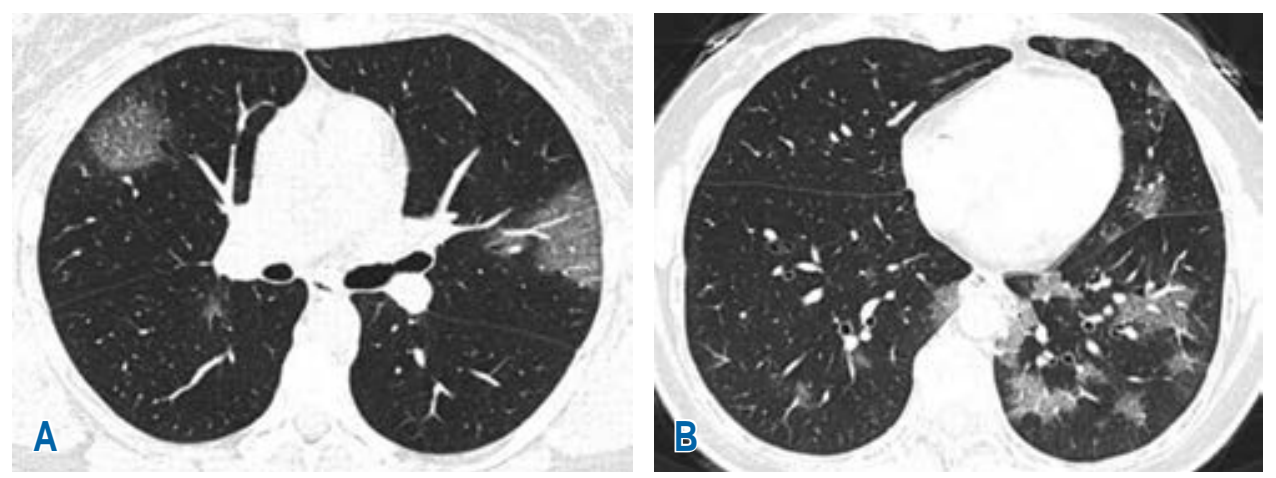

cional en 33\%, los destinos más visitados fueron ciudades de Europa, las cuales se encuentran en Italia y España.

El promedio de días del período de duración de la enfermedad a la solicitud de atención fue de ocho días (5). La fiebre estuvo presente en $91 \%$ (51) de los pacientes, seguida de tos, cefalea y disnea. La saturación de oxígeno al ingreso fue en promedio de $85 \%$, con $30 \%$ (17) de pacientes intubados (Tabla 1).

De acuerdo con los hallazgos tomográficos, el patrón predominante en 39\% (22) de los casos fue patrón mixto (Figura 1) caracterizado por patrón en vidrio deslustrado, combinado con zonas de consolidación, seguido de patrón empedrado (Figura 2) y patrón en vidrio deslustrado único (Figura 3) con $30 \%$ (17) y $29 \%$ (16), respectivamente. La localización de las lesiones fue bilateral en 88\% (52) de los casos estudiados con mayor involucro de lóbulos inferiores en 55\% (31), seguido de afectación en lóbulos superiores en 36\% (20). La distribución de las lesiones fue predominantemente subpleural en $48 \%$ (27) de los casos estudiados (Figura 4), seguida de afectación con distribución en parches en 20\% (11) (Figura 5). En otros hallazgos predominaron las linfadenopatías con 25\% (14), seguidas de las atelectasias en 23\% (13) (Tabla 2).

Se realizó análisis comparativo de las variables clínicas y sociodemográficas de acuerdo con el patrón mixto y otros patrones sin identificar diferencias significativas en la población estudiada (Tabla 3).

\section{DISCUSIÓN}

El objetivo de nuestro estudio fue describir los hallazgos tomográficos de la afectación pulmonar por COVID-19 en la población mexicana, observándose predominio del patrón mixto, caracterizado por vidrio deslustrado y áreas de consolidación. Al contrastar nuestros resultados con lo descrito, encontramos que Pan et al. demostraron que los hallazgos tomográficos fueron variables acorde a la evolución de la enfermedad, evidenciando en la segunda etapa de la enfermedad (entre cinco y ocho días) mayor extensión del patrón en vidrio deslustrado en $82 \%$ de los pacientes, asociado a un patrón empedrado en 53\% y consolidaciones en $47 \%$. Se evidenció correlación con los hallazgos tomográficos en nuestro estudio donde el día promedio de la enfermedad ocho es correspondiente a la segunda etapa de la enfermedad, lo que refleja su progresión. ${ }^{8}$ Dada la evaluación única del estudio inicial de los pacientes sería relevante considerar el seguimiento de la enfermedad.

Las lesiones tomográficas pulmonares se encontraron con mayor frecuencia de forma bilateral, de distribución subpleural y en lóbulos inferiores, concordando con el metaanálisis realizado por Salehi et al., el cual reflejó afectación bilateral y periférica en 87.5 y $76 \%$, respec-

Tabla 2. Hallazgos en imagen por tomografía computarizada en casos con sospecha de COVID-19.

\begin{tabular}{|l|c|}
\hline Variables & $\mathrm{n}=56$ \\
\hline Patrón tomográfico & \\
Opacidades en vidrio deslustrado & $16(29)$ \\
Consolidación & $1(2)$ \\
Empedrado & $17(30)$ \\
Mixto & $22(39)$ \\
\hline Localización & \\
Unilateral & $4(12)$ \\
Bilateral & $52(88)$ \\
\hline Afectación por lóbulo & \\
Superior & $20(36)$ \\
Medio o língula & $3(5)$ \\
Inferior & $31(55)$ \\
Todos & $2(4)$ \\
\hline Distribución & \\
Subpleural & $27(48)$ \\
Peribroncovascular (central) & $9(16)$ \\
Subpleural-peribroncovascular & $9(16)$ \\
Parches & $11(20)$ \\
\hline Otros hallazgos & \\
Linfadenopatía & $14(25)$ \\
Atelectasia & $13(23)$ \\
Nódulos & $5(9)$ \\
\hline
\end{tabular}

Variables cualitativas expresadas en frecuencia (\%). 
tivamente, con mayor involucro de lóbulos inferiores. ${ }^{7}$ Además, el estudio de Wang et al. que reportó 85\% de afectación bilateral de 90 pacientes valorados en su estudio, también encontró afectación predominante subpleural posterior al inicio de los síntomas, variando de $63 \%$ a $73 \%$ en el transcurso de los días de la enfermedad. ${ }^{13}$ Dado que nuestro estudio refleja la valoración inicial de los hallazgos, lo cual podría ser una limitante, se considera de suma importancia el seguimiento progresivo del comportamiento de las lesiones. ${ }^{13}$

Otros hallazgos asociados como linfadenopatías, cavitaciones o derrames pleurales no son hallazgos frecuentes descritos en la neumonía por coronavirus. ${ }^{6}$ En nuestros pacientes se detectaron linfadenopatías, atelectasias, nódulos y engrosamiento peribroncovascular como hallazgos asociados. Salehi et al. reportaron hallazgos no comunes asociados en aproximadamente $7 \%$, cifra mayor que la

Tabla 3: Comparación de variables sociodemográficas y clínicas entre patrón mixto y otros patrones en pacientes con diagnóstico de COVID-19.

\begin{tabular}{|c|c|c|c|}
\hline \multirow[b]{2}{*}{ Variables } & \multicolumn{2}{|c|}{ Patrones } & \multirow[b]{2}{*}{$p$} \\
\hline & $\begin{array}{l}\text { Mixto } \\
\mathrm{n}=22\end{array}$ & $\begin{array}{l}\text { Otros } \\
n=34\end{array}$ & \\
\hline \multicolumn{4}{|l|}{$\begin{array}{l}\text { Características } \\
\text { sociodemográficas }\end{array}$} \\
\hline $\begin{array}{l}\text { Edad (años) } \\
\text { mediana (rango) }\end{array}$ & $49 \pm 15$ & $52 \pm 12$ & 0.33 \\
\hline Masculino & $13(59)$ & $21(62)$ & 0.84 \\
\hline $\begin{array}{l}\text { Presencia de } \\
\text { comorbilidades } \\
\text { *Tipo de comorbilidades }\end{array}$ & $9(41)$ & $20(59)$ & 0.19 \\
\hline - Diabetes mellitus & $4(18)$ & $14(41)$ & 0.06 \\
\hline $\begin{array}{ll}\text {. Hipertensión } \\
\text { arterial } \\
\text {. Obesidad }\end{array}$ & $\begin{array}{l}3(14) \\
1(5)\end{array}$ & $\begin{array}{l}8(24) \\
5(15)\end{array}$ & $\begin{array}{l}0.29 \\
0.23\end{array}$ \\
\hline \multicolumn{4}{|l|}{$\begin{array}{l}\text { Características de } \\
\text { la enfermedad }\end{array}$} \\
\hline $\begin{array}{l}\text { Duración de } \\
\text { enfermedad (días), } \\
\text { mediana (rangos) }\end{array}$ & $8 \pm 3$ & $8 \pm 6$ & 0.68 \\
\hline Fiebre & $20(91)$ & $31(91)$ & 0.66 \\
\hline Tos & $14(64)$ & $28(82)$ & 0.11 \\
\hline Cefalea & $14(64)$ & $24(71)$ & 0.58 \\
\hline Disnea & $13(62)$ & $21(64)$ & 0.89 \\
\hline Dolor pleurítico & $2(9)$ & $5(15)$ & 0.53 \\
\hline $\begin{array}{l}\text { Saturación de oxígeno } \\
(\%) \text {, mediana (rangos) }\end{array}$ & $84 \pm 75$ & $86 \pm 64$ & 0.40 \\
\hline Intubado & $8(36)$ & $9(27)$ & 0.47 \\
\hline
\end{tabular}

encontrada en nuestra población, lo que refleja hallazgos en común con nuestro estudio.

Además, el derrame pleural no se identificó en nuestro estudio, lo cual podría estar relacionado al tiempo de evolución de la enfermedad considerando lo reportado por Wang et al. donde $50 \%$ de los pacientes que presentaron derrame pleural fueron en los días 11, 21 y 24 de la evolución de la enfermedad..$^{13}$

Nuestro estudio tiene algunas limitaciones inherentes al diseño del estudio, por lo tanto, se requieren estudios longitudinales para poder valorar la evolución de patrones tomográficos así como estudios con mayor número de pacientes.

Las fortalezas del estudio es la identificación de patrones tomográficos en la población mexicana, ya que son pocas las publicaciones que evalúan estas variables, lo que nos permite brindar a todo el personal de salud del territorio mexicano que está al frente de los servicios de imagen en la actual pandemia, información concisa sobre el espectro radiológico de la afectación pulmonar por COVID-19.

\section{CONCLUSIONES}

El comportamiento tomográfico de la población mexicana estudiada con afectación pulmonar por COVID-19 se caracterizó por dos patrones principales: tipo mixto (áreas combinadas de vidrio deslustrado y consolidación del espacio aéreo) y patrón en empedrado, característicos de la etapa progresiva de la enfermedad (segunda etapa clínica). Como institución de referencia nacional y hospital destinado a atención exclusiva COVID-19 es meritorio mencionar que el INER es hasta el momento, y será probablemente, la institución de salud con mayor concentración de casos con enfermedad avanzada y por ende, posibles pacientes en condiciones graves, lo cual podría estar íntimamente relacionado con los hallazgos hasta ahora reflejados en el presente estudio, dando pie a la necesidad de seguimiento mediante futuros estudios longitudinales.

La caracterización tomográfica de las lesiones pulmonares en cuanto a distribución subpleural, bilateralidad y mayor afectación en lóbulos inferiores constituyen hallazgos típicos de la enfermedad pulmonar por SARS-CoV-2 hasta ahora descritos en la literatura y estudios previos.

La tomografía de tórax es el método de imagen de elección para el diagnóstico, pronóstico y seguimiento de la enfermedad en estudio.

\section{REFERENCIAS}

1. Zhou P, Yang X, Wang X, et al. A pneumonia outbreak associated with a new coronavirus of probable bat origin. Nature 2020;579(7798):270273. https://doi.org/10.1038/s41586-020-2012-7. 
2. World Health Organization. Director General's remarks at the media briefing on 2019-nCoV on 11 February 2020. Access date: 2020 April 23. Available from: https://www.who.int/dg/speeches/detail/ who-director-general-s-remarks-at-the-media-briefing-on-2019-ncovon-11-february-2020.

3. World Health Organization. Coronavirus disease (COVID-19) Situation dashboard. Access date: 2020 April 23. Available from: https://who. sprinklr.com/region/amro/country/mx. Updated April 23, 2020.

4. Weinstock MB, Echenique A, Russell JW, et al. Chest $X$-ray findings in 636 ambulatory patients with COVID-19 presenting to an urgent care center: a normal chest $X$-ray is no a guarantee. JUCM 2020; $13-18$.

5. Franquet T. Imaging of pulmonary viral pneumonia. Radiology 2011;260(1):18-39. https://doi.org/10.1148/radiol.11092149.

6. Jung HK, Lim S, Choe J, Choi S-H, Sung H, Do K-H. Radiographic and CT features of viral pneumonia. Radiographics 2018;38(3):719739. https://doi.org/10.1148/rg.2018170048.

7. Salehi S, Abedi A, Balakrishnan S, Gholamrezanezhad A. Coronavirus disease 2019 (COVID-19): A systematic review of imaging findings in 919. AJR Am J Roentgenol 2020:1-7. https://doi.org/10.2214/ aj.20.23034.

8. Pan F, Ye T, Sun P, et al. Time course of lung changes on chest CT during recovery from coronavirus disease 2019 (COVID-19). Radiology 2020;295(3):715-721. doi: 10.1148/radiol.2020200370.
9. Lu R, Zhao X, Li J, et al. Genomic characterization and epidemiology of 2019 novel coronavirus: implications for virus origins and receptor binding. Lancet 2020;395(10224):565-574. https://doi.org/10.1016/ s0140-6736(20)30251-8.

10. Xu Z, Shi L, Wang Y, et al. Pathological findings of COVID-19 associated with acute respiratory distress syndrome. Lancet Respir Med 2020;8(4):420-422. https://doi.org/10.1016/s22132600(20)30076-X.

11. Lee Ch-H, Chen R-F, Liu J-W, et al. Altered p38 mitogen-activated protein kinase expression in different leukocytes with increment of immunosuppressive mediators in patients with severe acute respiratory syndrome. J Immunol 2004;172(12):7841-7847. https:/l doi.org/10.4049/jimmunol.172.12.7841.

12. Hansell DM, Bankeir AA, MacMahon H, McLoud TC, Müller NL, Remy J. Fleischer society: glossary of terms for thoracic imaging. Radiology 2008; 246(3):697-722. https://doi.org/10.1148/radiol.2462070712

13. Wang $\mathrm{Y}$, Dong $\mathrm{Ch}, \mathrm{Hu} \mathrm{Y}$, et al. Temporal changes of CT findings in 90 patients with COVI-19 pneumonia: a longitudinal study. Radiology 2020;200843. https://doi.org/10.1148/radiol.2020200843.

Conflicto de intereses: Los autores declaran no tener conflicto de intereses. 